This item was submitted to Loughborough's Research Repository by the author.

Items in Figshare are protected by copyright, with all rights reserved, unless otherwise indicated.

\title{
A numerical study on influence of strain gradients on lattice rotation in micro- machining of a single crystal
}

\section{PLEASE CITE THE PUBLISHED VERSION}

https://doi.org/10.20528/cjsmec.2021.03.001

\section{PUBLISHER}

Tulpar Academic Publishing

VERSION

VoR (Version of Record)

\section{PUBLISHER STATEMENT}

This is an Open Access Article. It is published by Tulpar Academic Publishing under the Creative Commons Attribution 4.0 Unported Licence (CC BY). Full details of this licence are available at: http://creativecommons.org/licenses/by/4.0/

\section{LICENCE}

CC BY 4.0

\section{REPOSITORY RECORD}

Demiral, Murat, Anish Roy, and Vadim Silberschmidt. 2021. "A Numerical Study on Influence of Strain Gradients on Lattice Rotation in Micro-machining of a Single Crystal”. Loughborough University. https://hdl.handle.net/2134/16697284.v1. 


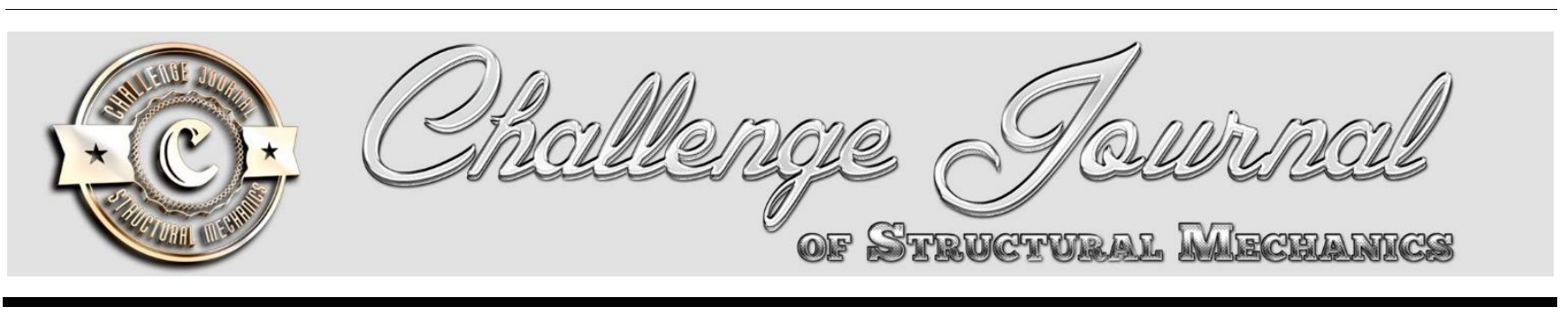

\title{
Research Article
}

\section{A numerical study on influence of strain gradients on lattice rotation in micro-machining of a single crystal}

\author{
Murat Demiral $^{\mathrm{a}, *}$ (D) Anish Roy ${ }^{\mathrm{b}}$ (D) , Vadim V. Silberschmidt ${ }^{\mathrm{b}}$ (D) \\ ${ }^{a}$ Department of Mechanical Engineering, University of Turkish Aeronautical Association, 06790 Ankara, Turkey \\ ${ }^{\mathrm{b}}$ Wolfson School of Mechanical, Electrical and Manufacturing Engineering, Loughborough University, LE11 3TU, UK
}

\section{ABSTRACT}

In latest years small scale machining has been widely used in advanced engineering applications such as medical and optical devices, micro- and nano-electro-mechanical systems. In micromachining of metals, a depth of cut becomes usually smaller than an average crystal size of a polycrystalline structure; thus, the cutting process zone can be localized fully indoors of a single grain. Due to the crystallographic anisotropy, development of small scale machining models accounting for crystal plasticity are essential for a precise calculation of material removal under such circumstances. For this purpose, a 3D finite-element model of micro-cutting of a single grain was developed. A crystal-plasticity theory accounting for gradients of strain, implemented in ABAQUS/Explicit via a user-defined material subroutine VUMAT, was used in the computations. The deformation-induced lattice rotations in micro-cutting of a single crystal were analyzed extensively.
\end{abstract}

\section{ART I CLE INF O}

Article history:

Received 9 May 2021

Revised 8 June 2021

Accepted 28 June 2021

\section{Keywords:}

Strain-gradient crystal plasticity

Orthogonal micromachining

Single crystal

Lattice rotation

Finite element analysis

\section{Introduction}

In latest years small scale machining has become popular in manufacturing of components with sizes of submillimeter or smaller used in advanced engineering applications such as medical and optical devices, microand nano-electro-mechanical systems. This process diverges significantly from conventional machining as uncut chip thickness is usually smaller than the crystal size of the sample; hence, instantaneous removal of the material takes place entirely within a crystal level. Since single crystals are strongly anisotropic in their mechanical behaviour, the cutting process naturally depends on crystallographic orientation as well as slip system and slip activity in single grain (Lee et al., 2000).

Deformation-induced lattice rotations, i.e. a texturesoftening factor, reorientation or lattice spin, have attracted attention in the micromachining community, since a close connection is present between crystallographic shear, the leading mechanism ruling the deformation, and the resulting texture evolution (Zaafarani et al., 2006). A few researches characterized the observed phenomena using various techniques including non-destructive 3D electron backscattered diffraction (Lee et al., 2000), transmission electron microscopy (Lloyd et al., 2005) and 3D synchrotron diffraction method (Yang et al., 2004). Nahata et al. (2021) investigated the subsurface microstructure in terms of the lattice rotation, recrystallization and shear bands after micromachining of aluminum single crystal experimentally. Lee and Zhou (1993) accounted for the texture softening factor in their analytical micro plasticity model to understand chip formation in micromachining.

Due to intrinsic inhomogeneity of deformation field in small scale machining tests, evaluating the obtained data is not simple. The computational methods are often used alternatively to understand its underlying mechanics. For instance, Zahedi et al. (2013) used a 3D combined finite element (FE)-smoothed particle hydrodynamics crystal plasticity model to study the influence of crystallography in machining of a copper single crystal. Similarly, Wang et al. (2020) developed a 2D crystal plasticity FE model to investigate the chip profile and shear angle, as well as their dependence on crystallography. Demiral

*Corresponding author. Tel.: +90-312-589-6112 ; E-mail address: mdemiral@thk.edu.tr (M. Demiral)

ISSN: 2149-8024 / DOI: https://doi.org/10.20528/cjsmec.2021.03.001 
et al. (2014a) investigated the effect of grain orientation on the behavior of a single crystal using a FE model incorporating the strain-gradient and crystal plasticity in the constitutive equations. However, in none of the above studies, the texture evolution during micromachining of a single crystal was studied comprehensively.

In this study, a three dimensional finite-element study of micro-machining of a b.c.c. single crystal is performed. The role of strain gradients due to inhomogeneous plastic deformation in small scales on the response of the structure cannot be ignored; thus, they are considered in the constitutive equations. Their effects on the spin of the crystalline lattice and resulting deformation patterns are presented.

This paper is divided into five sections. Section 2 introduces the constitutive equations of the theory used in the simulations. Section 3 describes the details of the developed FE model. Section 4 shows the obtained results with their associated discussions. Section 5 presents the concluding remarks of the study.

\section{Theory}

In the simulations, an enhanced modelling scheme for a strain-gradient crystal-plasticity (EMSGCP) theory reported in Demiral et al. (2017) was used. In the following, a dot superposed on a symbol indicates a material time derivative and a bold symbol denotes a vector or a tensor. The constitutive equations are summarized as follows:

Elastic $\left(F^{e}\right)$ and plastic $\left(F^{p}\right)$ parts are the components of the deformation gradient $F$ according to Eq. (1).

$F=F^{e} F^{p}$

The following flow equation rules the evolution of $F^{p}$

$\dot{F}^{p}=L^{p} F^{p}$

where $L^{p}$, the plastic velocity gradient, is expressed as

$L^{p}=\sum_{\alpha=1}^{N} \dot{\gamma}^{\alpha} S^{\alpha} \otimes m^{\alpha}$

In Eq. (3), $\dot{\gamma}^{\alpha}$ is the shearing rate on the slip system $\alpha$. $s^{\alpha}$ and $m^{\alpha}$ are the slip direction and the slip-plane normal, respectively. A power-law representation is used for $\dot{\gamma}^{\alpha}$, as

$\dot{\gamma}^{\alpha}=\dot{\gamma}_{0}^{\alpha} \operatorname{sgn}\left(\tau^{\alpha}\right)\left|\frac{\tau^{\alpha}}{g_{T}^{\alpha}}\right|^{n}$

Here $\dot{\gamma}_{0}^{\alpha}$ is the reference strain rate, $\tau^{\alpha}$ is the resolved shear stress, $n$ is the material constant related to its ratesensitivity, $g_{T}^{\alpha}$ represents the strength of the slip system $\alpha$ at the instant time, and $\operatorname{sgn}(\boldsymbol{\Gamma})$ is the signum function of $\boldsymbol{\Gamma}$.

In this model, the critical resolved shear stress $\left(\left.g_{T}^{\alpha}\right|_{t=0}\right)$ is ruled by the initial strength of slip systems associated with statistically stored densities (SSDs) $\left(\left.g_{s}^{\alpha}\right|_{t=0}\right)$ and ge- ometrically necessary dislocations (GNDs) $\left(\left.g_{G}^{\alpha}\right|_{t=0}\right)$. They are related to initial SSD $\left(\left.\rho_{S}\right|_{t=0}\right)$ and GND $\left(\left.\rho_{G}\right|_{t=0}\right)$ densities, respectively, via the constant, $K$ :

$\left.g_{T}^{\alpha}\right|_{t=0}=\sqrt{\left(\left.g_{S}^{\alpha}\right|_{t=0}\right)^{2}+\left(\left.g_{G}^{\alpha}\right|_{t=0}\right)^{2}}$

$\left.g_{S}^{\alpha}\right|_{t=0}=K \sqrt{\left.\rho_{s}\right|_{t=0}}$

$\left.g_{G}^{\alpha}\right|_{t=0}=K \sqrt{\left.\rho_{G}\right|_{t=0}}=K \sqrt{\left.\rho\right|_{t=0}(\overline{S / V})^{2}}$

The GND density term considered the normalized surface-to-volume $(\overline{S / V})$ ratio of the component (Demiral et al., 2017). The slip strength during loading progresses due to SSDs $\left(\Delta g_{S}^{\alpha}\right)$ and GNDs $\left(\Delta g_{G}^{\alpha}\right)$ on the slip system as follows:

$g_{T}^{\alpha}=\left.g_{T}^{\alpha}\right|_{t=0}+\sqrt{\left(\Delta g_{S}^{\alpha}\right)^{2}+\left(\Delta g_{P}^{\alpha}\right)^{2}}$

$\Delta g_{S}^{\alpha}=\sum_{\beta=1}^{N} h_{\alpha \beta} \Delta \gamma^{\beta}$

$\Delta g_{G}^{\alpha}=\alpha_{T} \mu \sqrt{b n_{G}^{\alpha}}$

where $h_{\alpha \beta}$ is the slip-hardening modulus, $\alpha_{\mathrm{T}}, \mu, b$ and $n_{G}^{\alpha}$ represent the Taylor coefficient, the modulus of shear, the Burgers vector and the effective density of GNDs, respectively. The following hardening model was used to calculate $h_{\alpha \beta}$ :

$h_{\alpha \beta}=q h_{\alpha \alpha}(\alpha \neq \beta)$

$h_{\alpha \alpha}=h_{0} \operatorname{sech}^{2}\left|\frac{h_{0} \tilde{\gamma}}{\left.g_{T}^{\alpha}\right|_{s a t}-\left.g_{T}^{\alpha}\right|_{t=0}}\right|$

$\tilde{\gamma}=\sum_{\alpha} \int_{0}^{t}\left|\dot{\gamma}^{\alpha}\right| d t$

where $h_{0}$ denotes the initial hardening parameter, $\tilde{\gamma}$ is the cumulative shear strain on all slip systems, $\left.g_{T}^{\alpha}\right|_{\text {sat }}$ is the saturation stress of the slip system $\alpha, q$ is the latent hardening ratio, considered to be 1 . The effective GND density $\left(n_{G}^{\alpha}\right)$ equals to:

$n_{G}^{\alpha}=\left|m^{\alpha} \times \sum_{\beta} s^{\alpha \beta} \nabla \gamma^{\beta} \times m^{\beta}\right|$

Here $s^{\alpha \beta}=s^{\alpha} . s^{\beta}$ and $\nabla \gamma^{\beta}$ represents the gradient of shear strain for each slip system. In an enhanced model of crystal-plasticity (EMCP), $\Delta g_{G}^{\alpha}$ disappears as it does not consider the evolving GNDs. The models were implemented in the FE software ABAQUS/Explicit via the userdefined material subroutine (VUMAT) (Demiral et al., 2016).

In this study, the micro-machining of a single crystal $\beta$-brass having a b.c.c. crystalline structure is investigated. The active slip systems for this structure are $\{110\}<111>$ (Ueda et al., 1980); thus, only this slip system set-among three potential system in b.c.c. materialswas activated in the computations. The respective planes and directions of the systems are presented in Table 1. Material constants and model parameters used in the simulations are given in Table 2. 
Table 1. The planes and directions of slip systems for $\{110\}<111>$ set

\begin{tabular}{ccc}
\hline System & Plane & Direction \\
\hline 1 & $\left(\begin{array}{lll}0 & 1 & 1\end{array}\right)$ & {$\left[\begin{array}{lll}1 & -1 & 1\end{array}\right]$} \\
2 & $\left(\begin{array}{lll}0 & 1 & 1\end{array}\right)$ & {$\left[\begin{array}{lll}1 & 1 & -1\end{array}\right]$} \\
3 & $\left(\begin{array}{llll}1 & 0 & 1\end{array}\right)$ & {$\left[\begin{array}{lll}-1 & 1 & 1\end{array}\right]$} \\
4 & $\left(\begin{array}{llll}1 & 0 & 1\end{array}\right)$ & {$\left[\begin{array}{lll}1 & 1 & -1\end{array}\right]$} \\
5 & $\left(\begin{array}{llll}1 & 1 & 0\end{array}\right)$ & {$\left[\begin{array}{lll}-1 & 1 & 1\end{array}\right]$} \\
6 & $\left(\begin{array}{llll}1 & 1 & 0\end{array}\right)$ & {$\left[\begin{array}{lll}1 & -1 & 1\end{array}\right]$} \\
7 & $\left(\begin{array}{llll}0 & -1 & 1\end{array}\right)$ & {$\left[\begin{array}{lll}1 & 1 & 1\end{array}\right]$} \\
8 & $\left(\begin{array}{llll}0 & -1 & 1\end{array}\right)$ & {$\left[\begin{array}{lll}-1 & 1 & 1\end{array}\right]$} \\
9 & $\left(\begin{array}{llll}1 & 0 & -1\end{array}\right)$ & {$\left[\begin{array}{lll}1 & 1 & 1\end{array}\right]$} \\
10 & $\left(\begin{array}{llll}1 & 0 & -1\end{array}\right)$ & {$\left[\begin{array}{lll}1 & -1 & 1\end{array}\right]$} \\
11 & $\left(\begin{array}{llll}-1 & 1 & 0\end{array}\right)$ & {$\left[\begin{array}{lll}1 & 1 & 1\end{array}\right]$} \\
12 & $\left(\begin{array}{lll}-1 & 1 & 0\end{array}\right)$ & {$\left[\begin{array}{lll}1 & 1 & -1\end{array}\right]$} \\
\hline
\end{tabular}

Table 2. Material and model parameters used in simulations

(Demiral et al., 2014a; Jona et al., 2001; Jia et al., 2012; Beyerlein et al., 2011).

\begin{tabular}{|c|c|c|c|c|c|}
\hline \multicolumn{3}{|c|}{ Elastic properties } & \multicolumn{3}{|c|}{ Density and damping parameters } \\
\hline$C_{11}(\mathrm{GPa})$ & $C_{12}(\mathrm{GPa})$ & $C_{44}(\mathrm{GPa})$ & $\rho\left(\mathrm{kg} \mathrm{m}^{-3}\right)$ & $\begin{array}{l}\text { Linear bulk } \\
\text { viscosity }\end{array}$ & $\begin{array}{l}\text { Quadratic bulk } \\
\text { viscosity }\end{array}$ \\
\hline 131.0 & 115.0 & 92.0 & 8500 & 0.06 & 1.2 \\
\hline \multicolumn{6}{|c|}{ Plastic properties } \\
\hline$\dot{\gamma}_{0}^{\alpha}\left(\mathrm{s}^{-1}\right)$ & $n$ & $q$ & $h_{0}(\mathrm{MPa})$ & $\left.g_{T}^{\alpha}\right|_{\text {sat }}(\mathrm{MPa})$ & $\alpha_{\mathrm{T}}$ \\
\hline $10^{-3}$ & 20 & 1 & 294 & 60 & 0.8 \\
\hline$K(\mathrm{MPa} \mathrm{mm})$ & $\mu(\mathrm{GPa})$ & $b(\mathrm{~mm})$ & $\left.\rho_{S}\right|_{t=0}\left(\mathrm{~mm}^{-2}\right)$ & $\left.\rho\right|_{t=0}\left(\mathrm{~mm}^{-2}\right)$ & $\overline{\overline{S / V}}$ \\
\hline 0.04 & 27.12 & $2.56 \times 10^{-7}$ & $6.810^{5}$ & $5.010^{4}$ & 4.36 \\
\hline
\end{tabular}

\section{Finite Element Modelling of Micro-cutting}

The FE modellings of structures have been widely used in the literature to investigate their responses when subjected to complex loading conditions (Ercan et al., 2015). Especially, the chip formation of a single crystal in microcutting process is multifaceted, where the crystal structure, crystal orientation and cutting direction play an important role in the formation of the chip. Its numerical model was developed. Details of the developed FE model simulating the orthogonal micro-cutting is shown in Fig. 1 . The dimensions of the modelled part of a single-crystal workpiece were: length $l=20 \mu \mathrm{m}$, height $h=20 \mu \mathrm{m}$ and width $w=0.48 \mu \mathrm{m}$. The sample was discretized using eight-node linear brick elements (C3D8) with a minimum element size of $60 \mathrm{~nm}$ in the process zone (resulted in 29600 elements). The rigid cutting tool was moving with a velocity of $1300 \mathrm{~mm} / \mathrm{s}$ in the cutting direction (CD, [abc] in Fig. 1) (Demiral et al., 2014a). Its rake and clearance angles are both $0^{\circ}$. While, the depth of cut $\left(a_{p}\right)$ equalled to $0.8 \mu \mathrm{m}$, the maximum cutting length was 1.20 $\mu \mathrm{m}$. They were selected to ensure the formation of a chip. An element-deletion technique available in ABAQUS/Explicit (2013) was employed in our cutting simulations. A failure criterion based on the Taylor cumulative shear strain with a threshold value of 3.0 (represents the onset of damage) was used to delete the elements (Demiral et al., 2014a). Friction between the contacting bodies was ignored in the simulations (Demiral et al., 2014b).

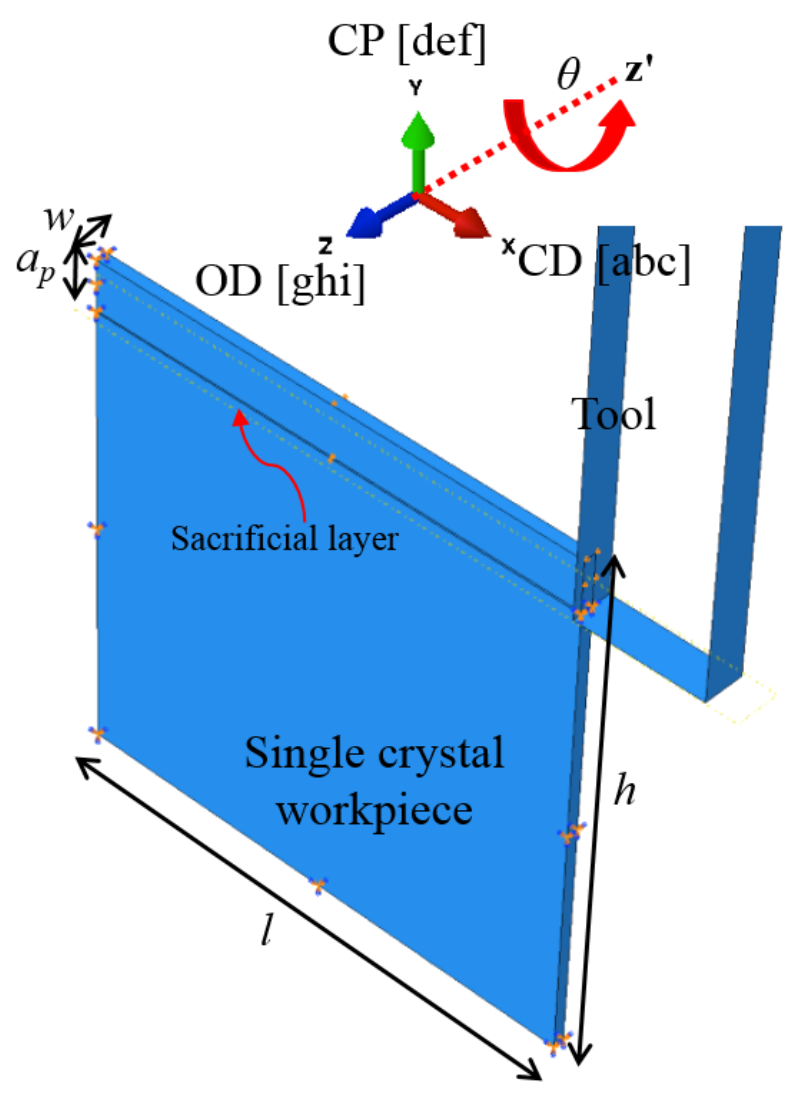

Fig. 1. Details of the developed FE model for micro-cutting of single-crystal $\beta$-brass. 


\section{Results and Discussion}

In this section, the results from the numerical computations of micro-cutting of single-crystal $\beta$-brass are presented. To evaluate the influence of rotation angle $\theta$ for a grain with the [101] axis aligned with the observation direction (OD, [ghi] in Fig. 1) on the lattice rotations, three cutting directions, namely $\theta=0^{\circ}, 35^{\circ}$ and $90^{\circ}$, were considered. The respective values are listed in Table 3 .

Table 3. Orientations CD ([abc] $)$ and CP ([def $]$ ) for various $\theta$ values for [ $\left.\begin{array}{lll}1 & 0 & 1\end{array}\right]$ axis aligned with the OD (see Fig. 1)

\begin{tabular}{cccc}
\hline$\theta$ & $0^{\circ}$ & $35^{\circ}$ & $90^{\circ}$ \\
\hline$[\mathrm{abc}]$ & {$\left[\begin{array}{lll}0 & -1 & 0\end{array}\right]$} & {$\left[\begin{array}{ccc}-0.990 & -2 & 0.990\end{array}\right]$} & {$\left[\begin{array}{lll}-1 & 0 & 1\end{array}\right]$} \\
{$[\mathrm{def}]$} & {$\left[\begin{array}{lll}-1 & 0 & 1\end{array}\right]$} & {$\left[\begin{array}{llll}-1 & 0.990 & 1\end{array}\right]$} & {$\left[\begin{array}{ll}0 & 0\end{array}\right]$} \\
\hline
\end{tabular}

Crystal reorientation in the workpiece material for the grain orientations analyzed is shown in Fig. 2. Here, lattice rotations about the OD-axis are compared. First, the lattice spin, i.e. induced lattice rotation, were distributed in the chip in a significantly different way for the cases investigated. It was observed that its magnitude was largest for $\theta=90^{\circ}$, reaching some $65^{\circ}$, and smallest for $\theta=0^{\circ}$ with a maximum value of around $25^{\circ}$. It was found that while at $\theta=35^{\circ}, 90^{\circ}$ the texture evolved in the chip in both positive and negative directions, at $\theta=0^{\circ}$ this occurred only in the negative direction. Dashed lines in Fig. 2 separate the regions with positive and negative lattice rotations in the deformed zone (representing the formation of a chip). In both orientations, for $\theta=35^{\circ}, 90^{\circ}$, crystal reorientation occurs in the opposite directions in the upper region of the chip and in the lower part. While the boundary between them is nearly vertical for $\theta=35^{\circ}$, it is more horizontal for $\theta=90^{\circ}$.

\section{Lattice Rotations $\left({ }^{\circ}\right)$}

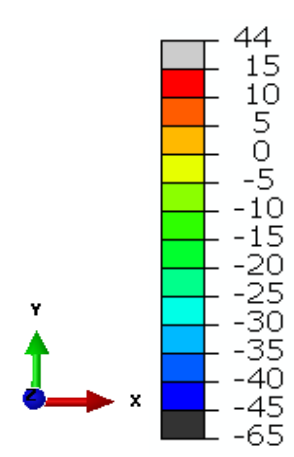

65

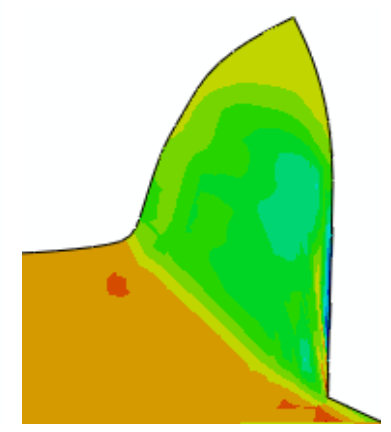

$\theta=0^{\circ}$

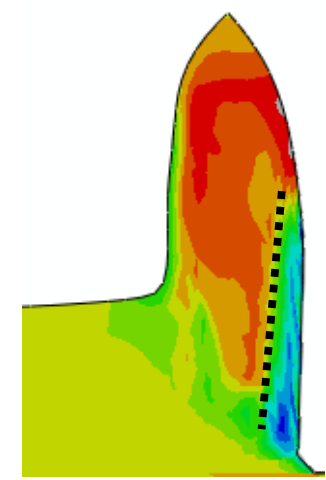

$\theta=35^{\circ}$

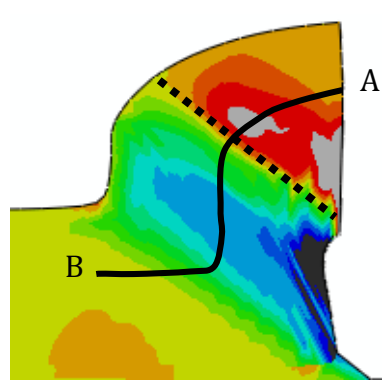

$\theta=90^{\circ}$

Fig. 2. Lattice rotation angles with their signs obtained by FE simulations of micro-machining at cutting length of $1.20 \mu \mathrm{m}$ for various crystallographic orientations (Dashed line separates the regions in the chip with positive and negative lattice rotations).

There is a tight link between crystallographic shear, the key mechanism leading the deformation through slip systems, and the resulting grain reorientation. In connection with this, to understand the differences in the characteristics of the lattice rotations depending on the rotation angle $\theta$, the role of activated slip systems was scrutinized. Fig. 3 presents a spatial 3D distribution of individual shear strain values on the active slip systems for $\theta=90^{\circ}$ of [ $\left[\begin{array}{lll}1 & 0 & 1\end{array}\right]$. It was observed that slip systems 2 , $5,8,9,10$ and 12 accommodated the overall shear in the workpiece, where the second/twelfth slip systems were the most active ones followed by the fifth/eighth systems and ninth/tenth systems in a row. The contributions of other slip systems were negligibly small; thus, they were not presented in Fig. 3.

The comparative individual contributions of various slip systems in the region of workpiece with higher shear activity ( $\tilde{\gamma}$ larger than 0.05 ) for different $\theta$ values are presented in Fig. 4. It was noticed that, there existed only 4 active slip systems for $\theta=0^{\circ}$ with 5 th and 8 th systems carried around $62.6 \%$ of the overall shearing while the 2nd and 12 th carried more than $30 \%$. When $\theta$ was changed into $35^{\circ}$, significant changes were noted. The most dominant slip systems for $\theta=0^{\circ}$, 5th and 8th, became less active with just $6.31 \%$ contribution for each, whereas the 9 th and 10 th systems took over their role involving in more than $50 \%$ of the total deformation. The activity of 2 nd and 12 th systems remained constant. Consequently, there were 6 slip systems ruling the deformation for $\theta=35^{\circ}$ as well as for $\theta=90^{\circ}$, as explained above. Overall, the 9 th and 10 th systems did not participate in ruling the deformation for $\theta=0^{\circ}$, whereas they were contributing significantly and non-negligibly for $\theta$ $=35^{\circ}$ and $90^{\circ}$, respectively. These two slip systems seemed to be responsible for the sign change of the lattice rotations for the orientations with non-zero rotation angles observed above. Furthermore, the difference in terms of the active slip systems for different cutting directions affected chip morphology as observed in Fig. 2 (Demiral et al., 2014a; 2016).

The calculated magnitudes of shear angle for the cases investigated were $44^{\circ}, 55^{\circ}$ and $38^{\circ}$ for $\theta=0^{\circ}, 35^{\circ}, 90^{\circ}$, respectively (see Fig. 2). In ultra-precision machining, a continuous chip formation and good surface finish can be achieved for a chip having a larger shear angle (Lee, 1990). In connection with this, $\theta=35^{\circ}$ is preferable 
among the cutting directions considered here in small scale cutting of brass single crystals for an enhanced surface finish.

FE simulations for $\theta=90^{\circ}$ were also performed using the EMCP theory. Compared to it, the EMSGCP theory exemplifies the involvement of strain gradients and their progress in an inhomogeneous cutting process. Lattice rotations along the path A-B in Fig. 2 is presented in Fig. 5 for the two theories. It was observed that the lattice spin had a larger variation on the chosen path when the EMCP theory was used. Since deformation-induced incompatibility in the lattice spin for a material point can be accommodated by GNDs in the EMSGCP theory, the distributions of lattice rotations are smoother when compared to that for the EMCP theory as expected for a physically reasonable strain-gradient theory (Niordson and Hutchinson, 2003; Demiral et al., 2014b).

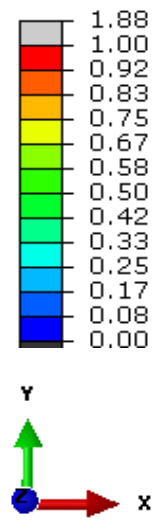

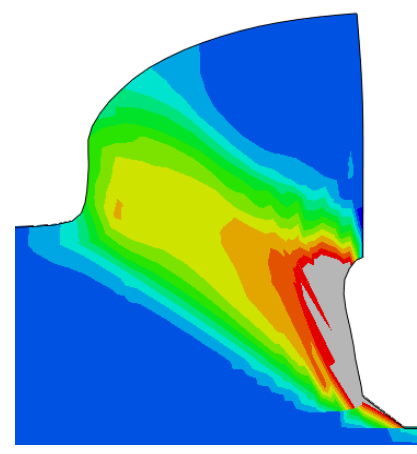

$2^{\text {nd }}$ and $12^{\text {th }}$

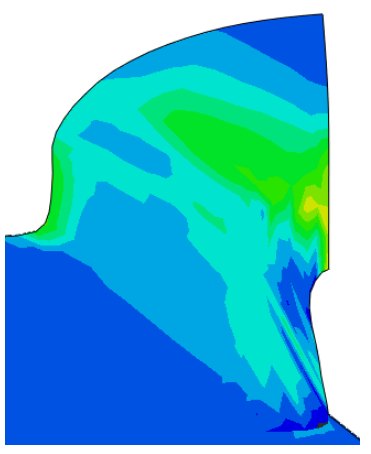

$5^{\text {th }}$ and $8^{\text {th }}$

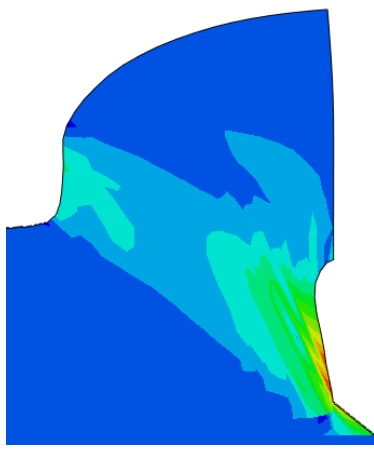

$9^{\text {th }}$ and $10^{\text {th }}$

Fig. 3. Distributions of shear strains for different active slip systems on the single crystal workpiece at cutting length of $1.2 \mu \mathrm{m}$ for $\theta=90^{\circ}$.

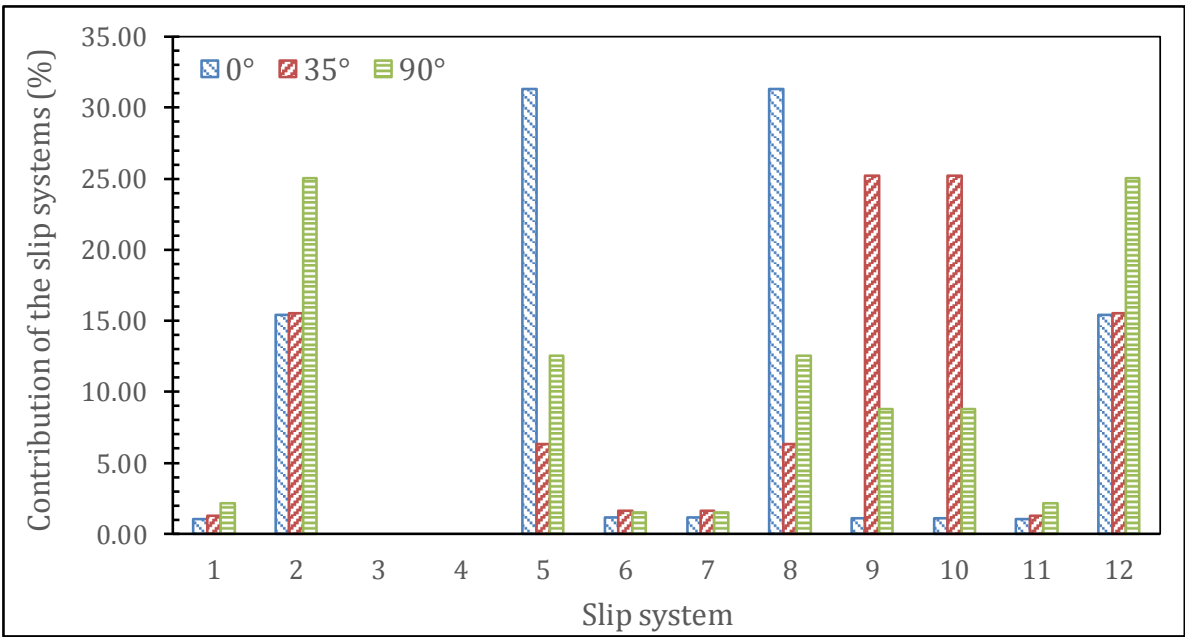

Fig. 4. Individual contributions of different slip systems (in \%) on the workpiece material points with $\tilde{\gamma}$ larger than 0.05 for different $\theta$ values.

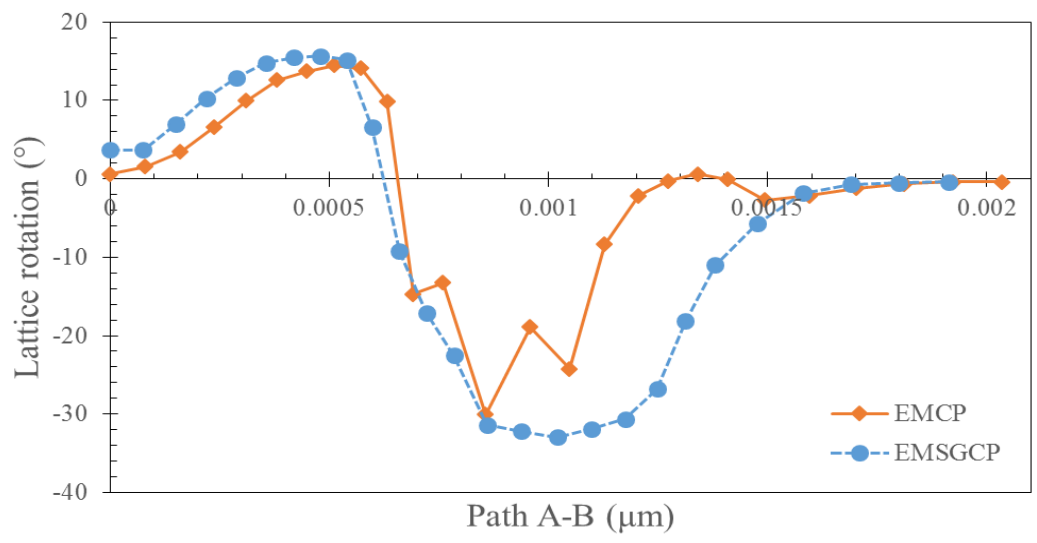

Fig. 5. Lattice rotation along path A-B in Fig. 2 at cutting length of $1.20 \mu \mathrm{m}$ for $\theta=90$ from numerical computations using different theories. 


\section{Conclusions}

The properties of final products are dependent on the resultant texture. Understanding the establishment and progress of deformation texture can provide theoretical insight for texture control, thus enhancing properties of the material. In connection with this, in this paper, the reorientation of crystals in the brass single crystal in micro-machining for different grain orientations was scrutinized using an advanced model. Our study confirmed the following conclusions:

- The patterns of lattice spins varied significantly for the cutting directions investigated. While both positive and negative lattice rotations in the chip were observed for $\theta=35^{\circ}, 90^{\circ}$, they were only negative for $\theta$ $=0^{\circ}$.

- The contributions of different slip systems were observed to be different for different orientations of a single crystal. That affected the magnitudes and sign change in the lattice spin as well as the resulting chip morphology.

- A smoother distribution of lattice spins was obtained using the EMSGCP compared to the EMCP theory.

In the future, the model will be further developed to investigate the texture evolution in micro-machining of polycrystalline samples.

\section{REFERENCES}

Beyerlein IJ, Mara NA, Bhattacharyya D, Alexander DJ, Necker CT (2011). Texture evolution via combined slip and deformation twinning in rolled silver-copper cast eutectic nanocomposite. International Journal of Plasticity, 27(1), 121-146.

Dassault Systemes (2013). Abaqus 6. 13 Analysis User Manual.

Demiral M, Roy A, El Sayed T, Silberschmidt VV (2014a). Influence of strain gradients on lattice rotation in nano-indentation experiments: A numerical study. Materials Science and Engineering: A, 608, 73-81.

Demiral M, Roy A, El Sayed T, Silberschmidt VV (2014b). Numerical modelling of micro-machining of fcc single crystal: influence of strain gradients. Computational Materials Science, 94, 273-278.

Demiral M, Roy A, Silberschmidt VV (2016). Strain-gradient crystalplasticity modelling of micro-cutting of bcc single crystal. Meccanica, 51(2), 371-381.

Demiral M, Nowag K, Roy A, Ghisleni R, Michler J, Silberschmidt VV (2017). Enhanced gradient crystal-plasticity study of size effects in a $\beta$-titanium alloy. Modelling and Simulation in Materials Science and Engineering, 25(3), 035013.

Ercan E, Alver N, Nuhoğlu A (2015). Evaluation of material properties by NDT methods and FEM analysis of a stone masonry arch bridge. Challenge Journal of Structural Mechanics, 1(4), 168-172.

Jia N, Roters F, Eisenlohr P, Kords C, Raabe D (2012). Non-crystallographic shear banding in crystal plasticity FEM simulations: Example of texture evolution in $\alpha$-brass. Acta Materialia, 60(3), 1099-1115.

Jona F, Marcus PM (2001). Structural and elastic properties of $\beta$-brass. Journal of Physics: Condensed Matter, 13(23), 5507.

Lee WB (1990). Prediction of microcutting force variation in ultra-precision machining. Precision Engineering, 12(1), 25-28.

Lee W, To S, Cheung C (2000). Effect of crystallographic orientation in diamond turning of copper single crystals. Scripta Materialia, 42(10), 937-945.

Lee WB, Zhou M (1993). A theoretical analysis of the effect of crystallographic orientation on chip formation in micromachining. International Journal of Machine Tools and Manufacture, 33(3), 439-447.

Lloyd SJ, Castellero A, Giuliani F, Long Y, McLaughlin KK, Molina-Aldareguia JM, Clegg WJ (2005). Observations of nanoindents via crosssectional transmission electron microscopy: A survey of deformation mechanisms. Proceedings of the Royal Society A: Mathematical, Physical and Engineering Sciences, 461(2060), 2521-2543.

Nahata S, Moradi M, Picard YN, Kota N, Ozdoganlar OB (2021). Micromachining imposed subsurface plastic deformation in single-crystal aluminum. Materials Characterization, 171, 110747.

Niordson CF, Hutchinson JW (2003). On lower order strain gradient plasticity theories. European Journal of Mechanics-A/Solids, 22(6), 771-778.

Ueda K, Iwata K, Nakayama K (1980). Chip formation mechanism in single crystal cutting of $\beta$-brass. CIRP Annals, 29(1), 41-46.

Wang Z, Zhang J, Xu Z, Zhang J, Li G, Zhang H, Li Z, ul Hassan H, Fang F, Hartmaier A, Yan Y (2020). Crystal anisotropy-dependent shear angle variation in orthogonal cutting of single crystalline copper. Precision Engineering, 63, 41-48.

Yang W, Larson BC, Pharr GM, Ice GE, Budai JD, Tischler JZ, Liu W (2004). Deformation microstructure under microindents in singlecrystal $\mathrm{Cu}$ using three-dimensional x-ray structural microscopy. Journal of Materials Research, 19(1), 66-72.

Zaafarani N, Raabe D, Singh RN, Roters F, Zaefferer S (2006). Threedimensional investigation of the texture and microstructure below a nanoindent in a Cu single crystal using 3D EBSD and crystal plasticity finite element simulations. Acta Materialia, 54(7), 1863-1876.

Zahedi SA, Demiral M, Roy A, Silberschmidt VV (2013). FE/SPH modelling of orthogonal micro-machining of fcc single crystal. Computational Materials Science, 78, 104-109. 